\title{
Voltage-Based Hot-Spot Detection Method for Defective Cell in Photovoltaic Module Using Projector
}

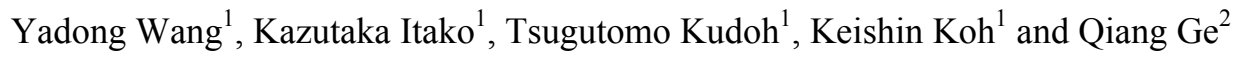 \\ 1. Department of Electrical and Electronic Engineering, Kanagawa Institute of Technology, Kanagawa 243-0292, Japan \\ 2. Department of Energy and Power Engineering, Yangzhou University, Yangzhou 225127, China
}

Received: May 12, 2016 / Accepted: May 26, 2016 / Published: August 31, 2016.

\begin{abstract}
This paper proposes a voltage-based hot-spot detection method for defective cells in PV module using projector. The presence of internal crystal defects is one of the main causes of hot-spot phenomenon in PV modules. Authors previously investigated the physical characteristics of hot-spot phenomenon referring to internal crystal defect. Based on it, a hot-spot detection method named as current-based SRC (self reverse current) detection method is developed. However, it becomes extraordinarily complicated to determine the defective cells under low illumination. In order to avoid this disadvantage, authors improve the SRC detection method by applying voltage. From the feasibility experiment results, it is confirmed that by calculating cell HSI (hotspots index) with voltage, the PV modules with defective cells can be prospectively excluded even under low illumination.
\end{abstract}

Key words: Hot-spot, PV module, crystal defect, detection method, hot spot index.

\section{Introduction}

Introduced in July 2012, Japan's FIT (feed-in tariff) policy was famously generous and triggered a surge in solar investment in the country. As PV installation density increases, more systems suffer from various failures. According to the failure reports, hot-spot phenomenon accounts for a large proportion [1].

A hot-spot consists of a localized overheating in PV modules. It appears when, due to some anomaly, such as a cracked or shadowed cell, the short circuit current of the affected cell becomes lower than the operating current of the whole, acting as an internal load, dissipating the power generated by other cells in the form of heat, while reducing the power output of the array [2]. Due to the poor thermal conductivities of cell encapsulation materials, temperatures of hundreds degrees centigrade are easily attained. Such temperatures degrade the optical and mechanical properties of encapsulation material, reducing array

Corresponding author: Kazutaka Itako, Ph.D., professor, research field: power electronics. performance and operating life [3]. In severe cases, the melting of interconnections can lead to total failure of the array [4]. Therefore, it is significant to inspect defective cells with high possibility of hot-spot phenomenon occurring before installation.

Currently, the conventional method of hot-spot inspection is thermography (infrared camera) [5]. Thermography is intended to discover the place where hot-spot phenomenon has occurred due to the temperature rise. Fig. 1 shows an IR (infrared radiation) image of one hot-spot. However, thermography inspection is susceptible to weather condition which is a great limitation. Furthermore, thermography consumes plenty of time, because, before shooting the IR pictures, it is necessary to add a shadow to the cell for a long time, respectively. Besides, a long time of appended shadows will be a large burden on the cells, and normal cells possibly deteriorate into hot-spot cells too. Meanwhile, experimental infrared cameras are quite expensive. Therefore, our research laboratory is striving to develop a new method for hot-spot inspection with the features of low cost and effort. 


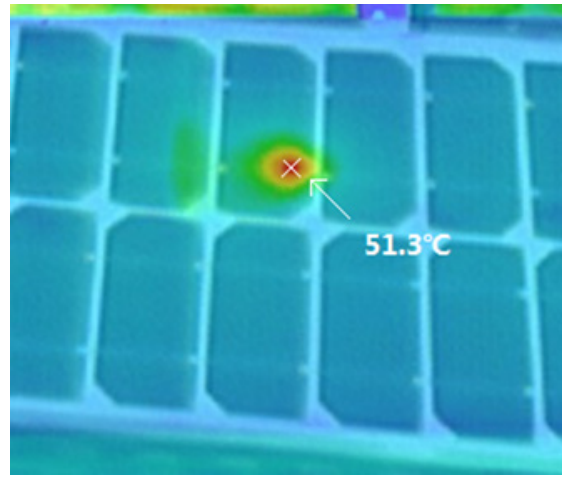

Fig. 1 IR Image of Hot-spot caused by crystal defect.

Authors previously investigated the hot-spot phenomenon causing by crystal defects, and developed a current-based SRC (self reverse current) detection method in the form of a projector which enables to diagnose defective cells $[6,7]$. However, when the cell surface illumination is low (under 2,000 Lux), it is difficult to determine the defective cell with the current-based SRC detection method.

Aiming to avoid this problem, authors propose an improved SRC detection method for PV modules, based on voltage. The effectiveness of this method is presented here.

\section{Theoretical Background}

Among the several causes of hot-spot phenomenon occurrence, our lab focus on the crystal defects which cause high surface recombination rate, appearing as high reverse leakage current of $p$ - $n$ junction when solar cells are partial shadowed. Its electrical characteristic has been investigated as follow.

By using a module which provides two terminals to each cell, Fig. 2 indicates the reverse leakage current value when $-10 \mathrm{~V}$ of DC supply is applied to each cell under light shielding state. This characteristic can substantially differ from one cell to another, even within the same module. The normal cells without defect exhibit a small reverse leakage current. Conversely, defective cells, with high possibility hot-spot arising, consequently present high reverse leakage current. The reason for this large leakage current will be explained as followed.

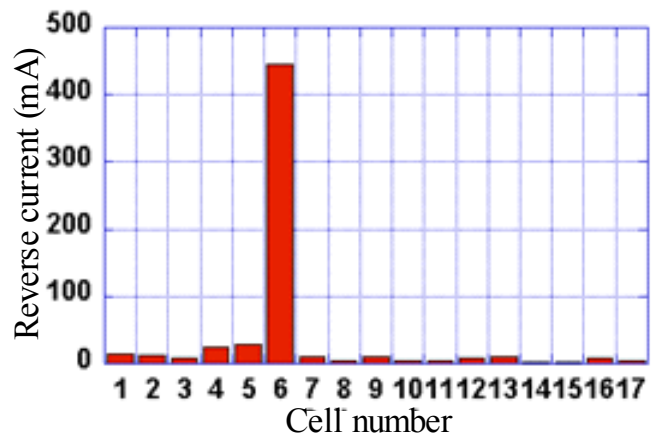

(a)

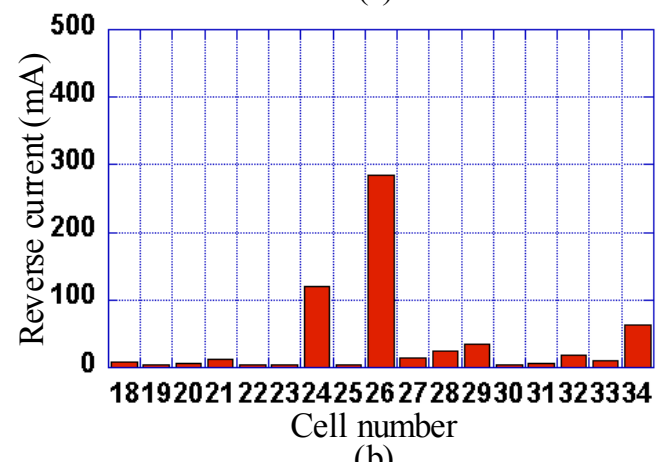

(b)

Fig. 2 Current value in each cell energized at $-10 \mathrm{~V}$.

Generally, the presence of crystal defects has two origins [8]. The first is the production of a crystal defect in the surface during manufacturing which results in high carrier recombination at the surface, the other reason is when a carrier is trapped and recombines in a crystal defect lattice within the semiconductor. The first case can be expressed by the following Eq. (1).

$$
\begin{gathered}
\mathrm{U}_{\text {Surf }}=\frac{p n-n_{i}{ }^{2}}{1 / S_{p}\left(n+n_{1}\right)+1 / S_{n}\left(p+p_{1}\right)} \\
\mathrm{S}_{\mathrm{p}}=\frac{1}{\tau_{\mathrm{p}} \mathrm{v}_{\mathrm{p}} \mathrm{N}_{\mathrm{t}}}, S_{n}=\frac{1}{\tau_{n} v_{n} N_{t}}
\end{gathered}
$$

where, $n_{i}$ is intrinsic density $\left(1 / \mathrm{cm}^{3}\right) ; S_{p}$ and $S_{n}$ are surface recombination velocity $(\mathrm{cm} / \mathrm{s}) ; \tau_{p}$ and $\tau_{n}$ are hole and electron lifetime (s); $v_{p}$ and $v_{n}$ are hole and electron thermal velocity $(\mathrm{cm} / \mathrm{s}) ; N_{t}$ is trap density $\left(1 / \mathrm{cm}^{3}\right) ; p_{1}$ and $n_{1}$ are hole and electron density when energy level $E_{t}$ of the recombination center and Fermi level $E_{f}$ are matched. The other case can be explained by Eq. (2).

$$
\mathrm{U}_{S R H}=\frac{p n-n_{i}^{2}}{\tau_{p}\left(n+n_{1}\right)+\tau_{n}\left(p+p_{1}\right)}
$$


Considering the theoretical formula for recombination, the reverse leakage current $J_{R}$ is given by the sum of the diode saturation current $J_{O}$ and the generation current $J_{G}$ produced by recombination of the carriers. It is described by Eq. (3).

$$
\begin{gathered}
J_{R}=J_{O}+J_{G} \\
J_{G}=\int q(-U) d x
\end{gathered}
$$

$U$ means total recombination rate that occurs in cells. According to Eqs. (1)-(3), when the carrier lifetime is shortened by crystal defects, the recombination rate increases and the leakage current component in the reverse direction rises [8]. By applying this physical phenomenon, a detection method named SRC detection is developed, and it enables to inspect the crystal defect cells in PV module.

\section{Current-Based SRC Detection Method and Its Limitation}

This section explains the specifications of experiment module and previously developed current-based SRC detection method. The illumination limitation of this method will also be mentioned in this section.

\subsection{Specification of Experimental Module}

Applied experimental PV solar module is exhibited in Fig. 3. This is a single-crystal type PV module without bypass diode. Thirty four cells are connected in one series. Table 1 shows the specification of it at $25{ }^{\circ} \mathrm{C}$, AM 1.5, $1000 \mathrm{~W} / \mathrm{m}^{2}$.

\subsection{Current-Based SRC Detection Method}

The current-based SRC detection system configuration is shown in Fig. 4. It is consisted of a projector, a PV module and the developed electronic control device. The projector is used to produce the shadowed and illuminated regions on the surface of the PV solar module. The electronic control device is responsible to measure the short circuit current from the PV module and control the animation.

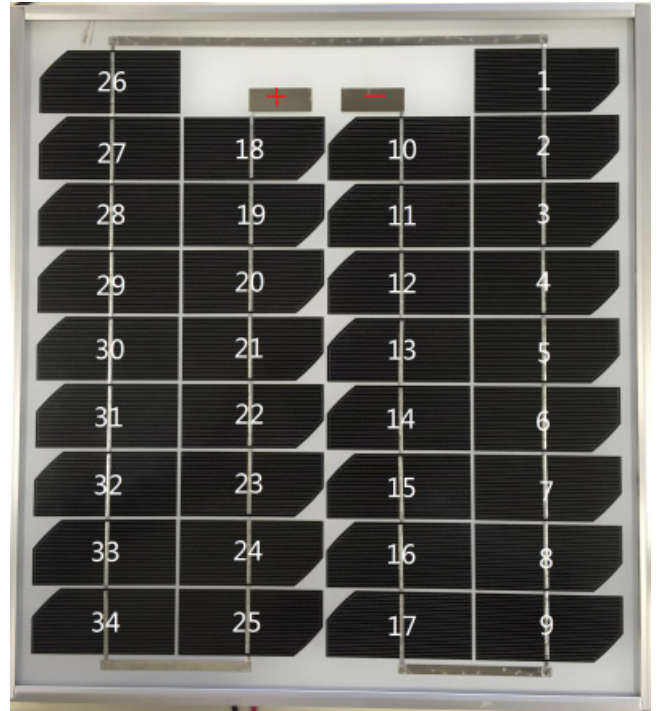

Fig. 3 Experimental module configuration.

Table 1 PV solar module specifications.

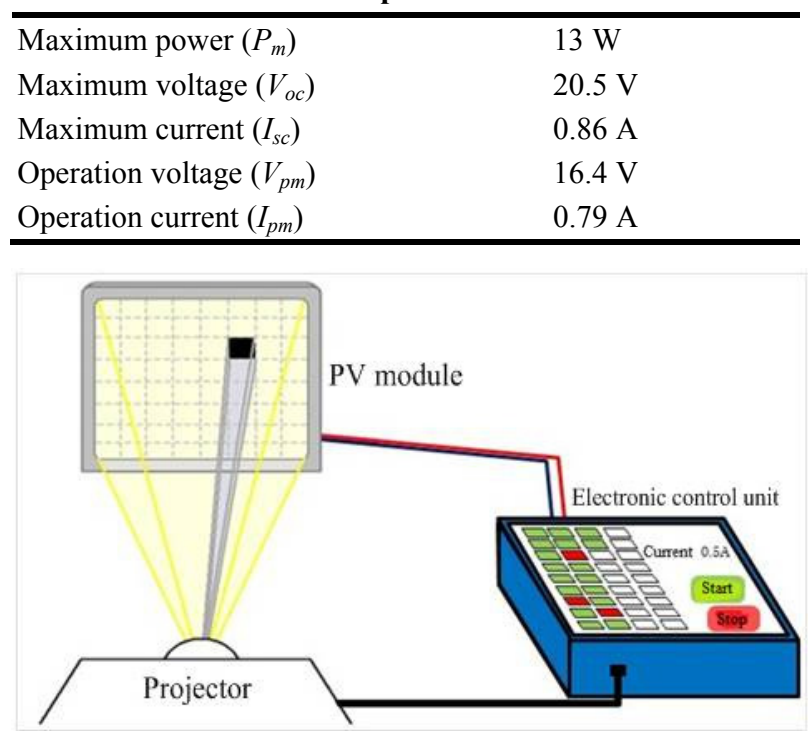

Fig. 4 Current-based SRC detection system configuration.

In this method, the index value that used to carry out the determination of the presence of hot-spots is defined as HSI (hot-spot index). First, as shown in Fig. $5 \mathrm{a}$, the initial current value $I$ is measured when the module is fully illuminated. In addition, as shown in Fig. $5 b$, each cell is partially shadowed in turn to get another current parameter $I_{s}$. The determination parameter HSI for defective cells is defined as Eq. (4). Experiments are conducted respectively under the illumination condition of 8,000 Lux and 2,000 Lux. Results are shown in Figs. 6 and 7. 


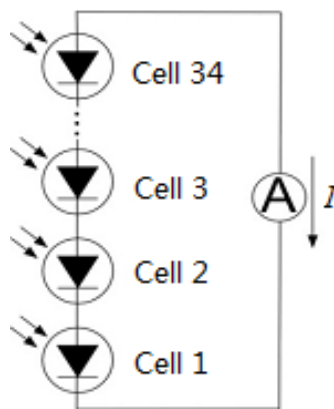

(a) No shadowing

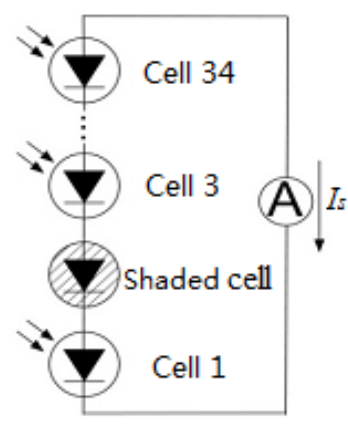

(b)Shadowing
Fig. 5 Experimental circuit configuration.

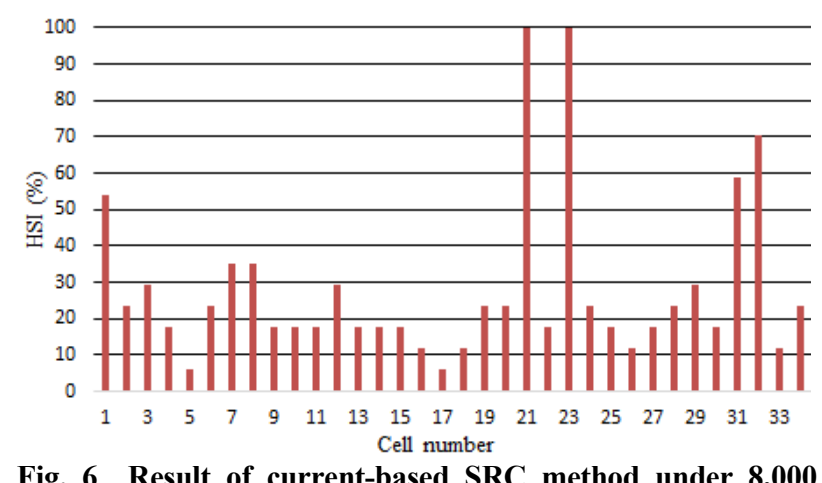

Fig. 6 Result of current-based SRC method under 8,000 Lux.

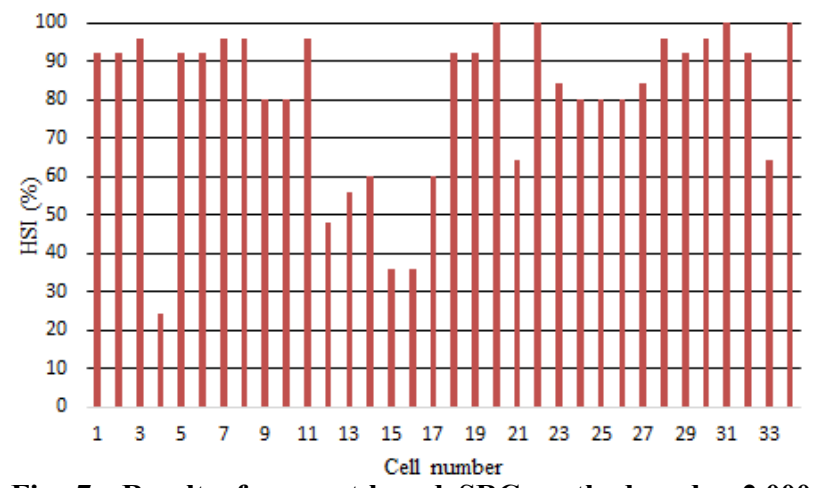

Fig. 7 Result of current-based SRC method under 2,000 Lux.

$$
\mathrm{HSI}=\frac{I_{S}}{I} \times 100 \%
$$

From the result under 8,000 Lux in Fig. 6, it is obvious that the HSI of No. 21, No. 23, No. 31 and No. 32 cells are relatively high. Therefore, these cells are high possibly hot-spot cells with defect. Nevertheless, from the result under the illumination of 2,000 Lux in Fig. 7, determination value of each cell is relatively high. It becomes extraordinarily confusing to determine the hot-spot cells. When simultaneously diagnose multiple solar modules in series, it is necessary to increase the distance between modules and projector. As a result, illuminance on the module surface will unavoidably drop, and so authors improve the SRC detection method by applying operating voltage.

\section{Proposed Voltage-Based SRC Detection Method}

Fig. 8 presents the $\mathrm{I}-\mathrm{V}$ characteristic of a solar module, with the decline of illumination, short-circuit current drops rapidly. Whereas the operating voltage is relatively stable. Therefore, the improved method applies operating voltage instead of short circuit current to diagnose defective cells.

\subsection{Detection Theory}

The equivalent circuit of voltage-based detection is shown in Fig. 9. Shaded normal cell share the same properties with a diode which reverse resistance is high, as well as the defective cell can be regarded as a low resistor. Generated power from illuminated cells energize at the shaded one.

Since the shaded normal cell becomes PN junction diodes with high electric resistance, the voltage share of the resistor $\mathrm{R}$ is low. Meanwhile, reverse bias resistance of the shaded defective cell is relatively low, so that the resistor $\mathrm{R}$ shares much higher voltage. Accordingly, the voltage ratio of the defective cell and resistor $\mathrm{R}$ are less susceptible to the illuminance. For this reason, it enables voltage-based SRC detection method to diagnose the defective cell at low illuminance.

\subsection{System Configuration}

Fig. 10 is the experimental system configuration for voltage-based SRC detection method. The experimental apparatus for detection consists of two units working simultaneously: the projector system for creating the shadowed and illuminated regions on the surface of the solar PV module and the measurement system to detect the voltage of matching resistor $\mathrm{R}$. 


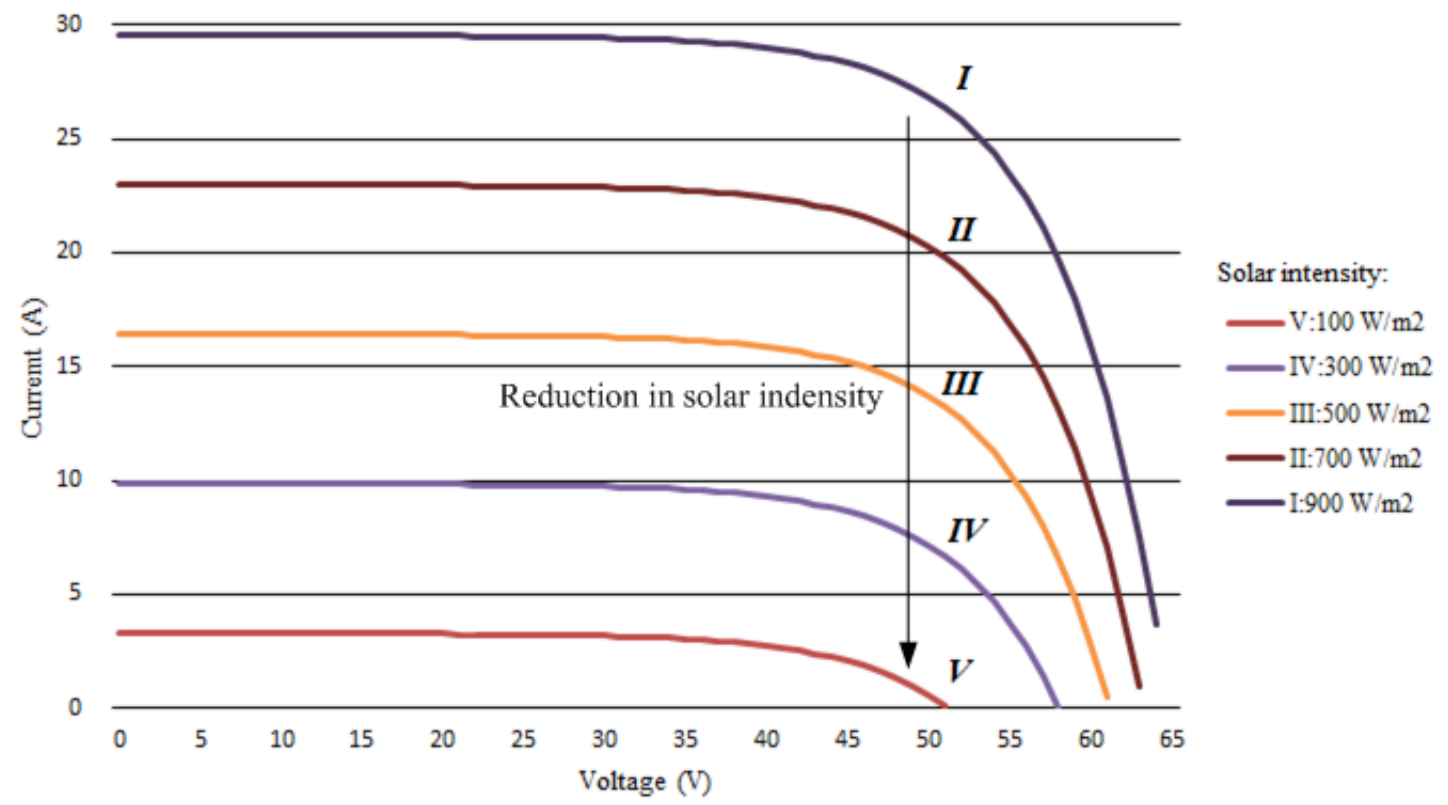

Fig. 8 The I-V characteristics of PV module vary with illuminance.
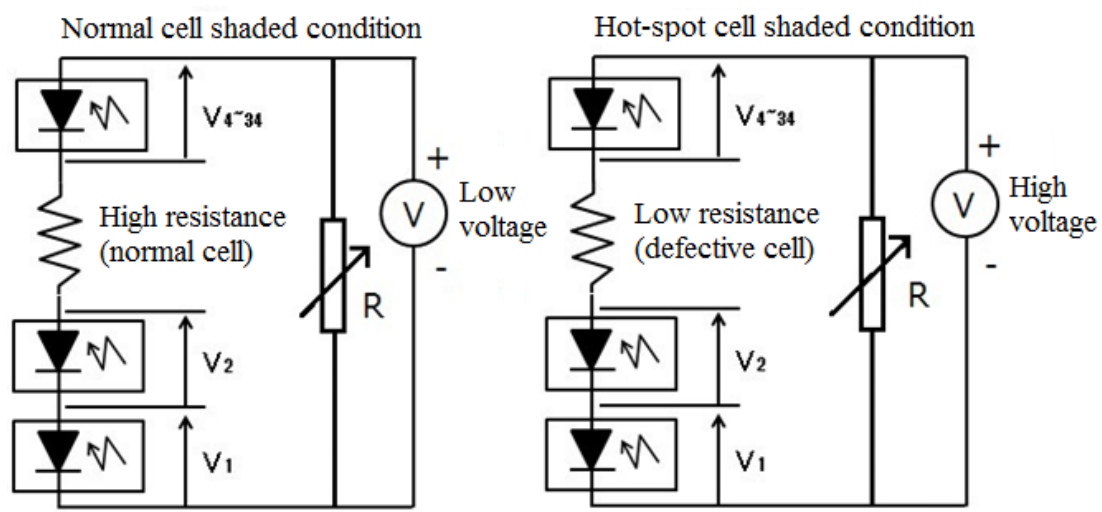

Fig. 9 Equivalent circuit of cell.

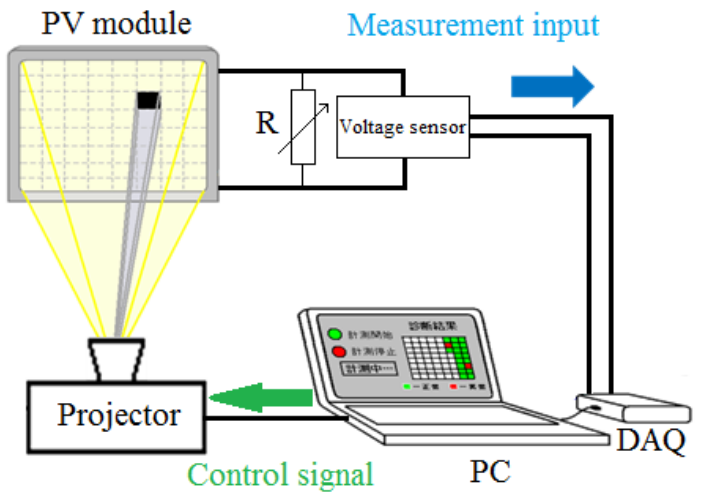

Fig. 10 Voltage-based SRC detection system configuration.

\subsection{Operating Principle}

The detection is started with illuminating the whole module by projector. Afterwards, variable resistor $\mathrm{R}$ is adjusted in order to achieve the maximum operating power. Then projector displays the animation as shown in Fig. 11.

The initial voltage value $V_{0}$ is measured when the module is fully illuminated as Step 1 in Fig. 11. Next, each cell is partially shadowed in turn to get another voltage parameter $V$. Therefore, the determination parameter HSI of voltage-based detection method is defined as following Eq. (5).

$$
H S I=\frac{V_{0}-V}{V_{0}} \times 100 \%
$$

According to the theory discussed in Section 4.1, if the HSI of the cell becomes extremely low it means this cell is high possibly defective. 


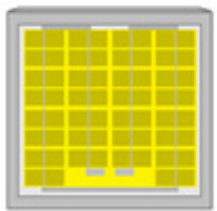

Step 1

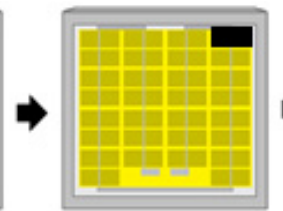

Step 2

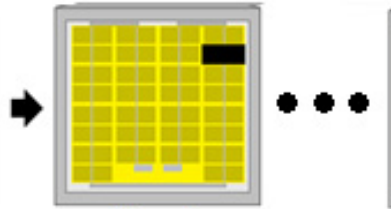

Step 3

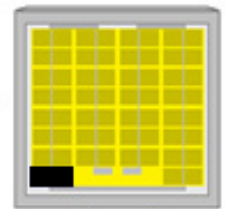

Step 35

Fig. 11 Animation operation of the detection.

\subsection{Resistance Setting}

Authors also investigated the influence of resistance on HSI. The characteristics of HSI for resistance R of a normal cell and a defective cell are indicated in Figs. 12 and 13.

From Fig. 12, it is obvious that the differences of HSI between the normal and defective cells decrease with an increase in the resistance value. In order to distinguish defective cells from normal cells clearly, the resistance should be slightly lower than matching resistor Rop. Meanwhile, due to Figs. 12 and 13, it

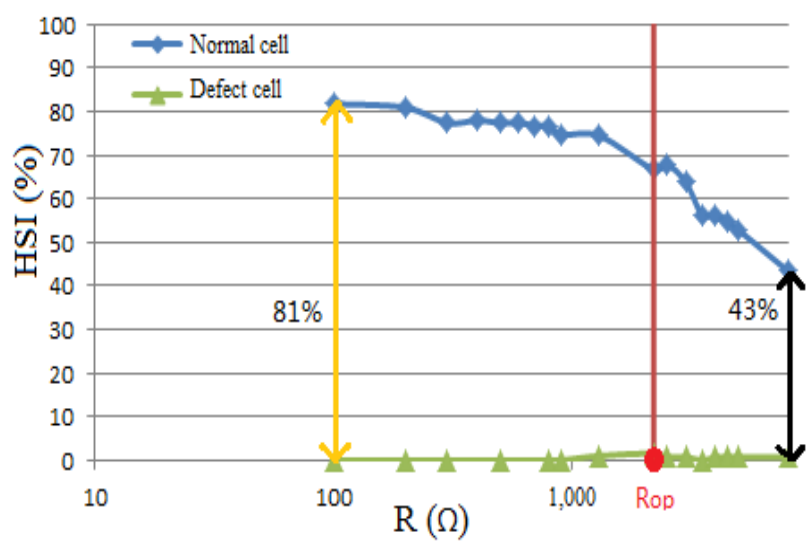

Fig. 12 Relations between HSI and resistance at 3,000 Lux.

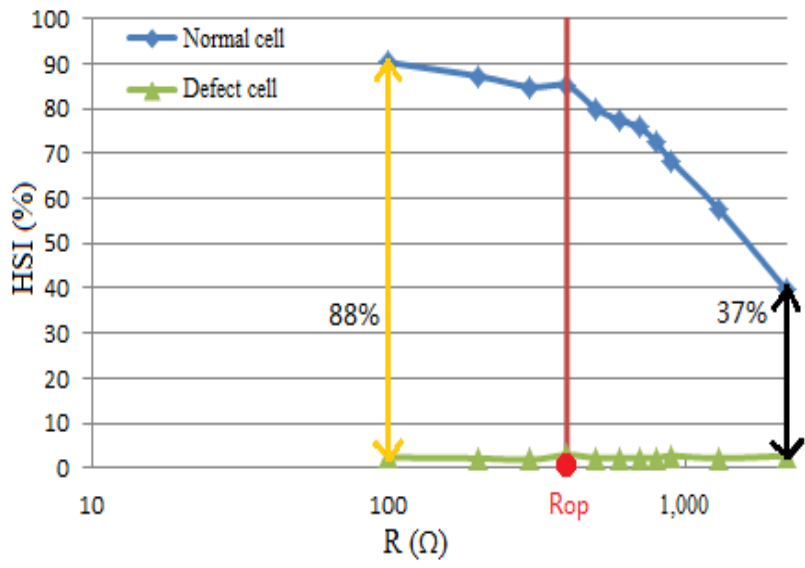

Fig. 13 Relations between HSI and resistance at 28,000 Lux. should be noted that the characteristic of HSI is also affected by illuminance. Therefore, it is necessary to change the resistance by the illumination.

\section{Experiment Results}

The PV solar module used is the same one used in current-based SRC detection experiment. Figs. 14 and 15 provide plots of a voltage-based SRC detection result, measured at illumination of 28,000 Lux and 2,000 Lux. The matching resistor $\mathrm{R}$ used is $3,600 \Omega$ which is $80 \%$ of Rop.

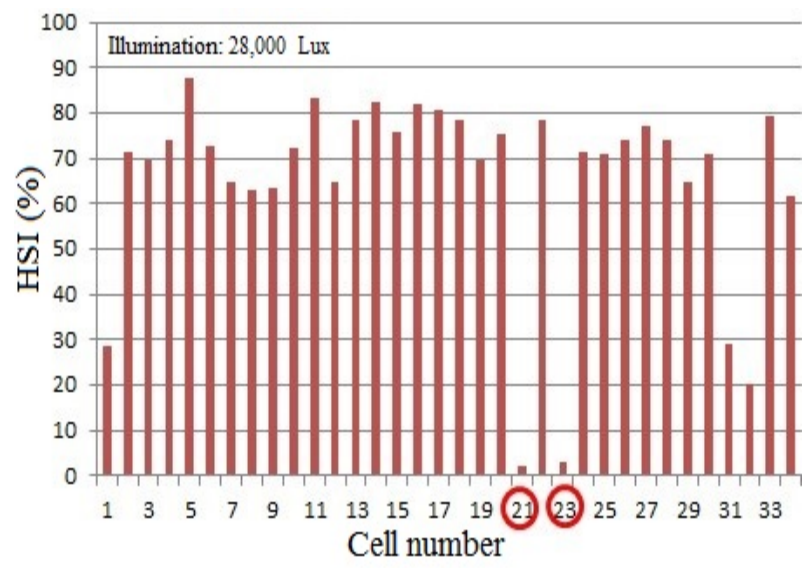

Fig. 14 Detection result at 28,000 Lux.

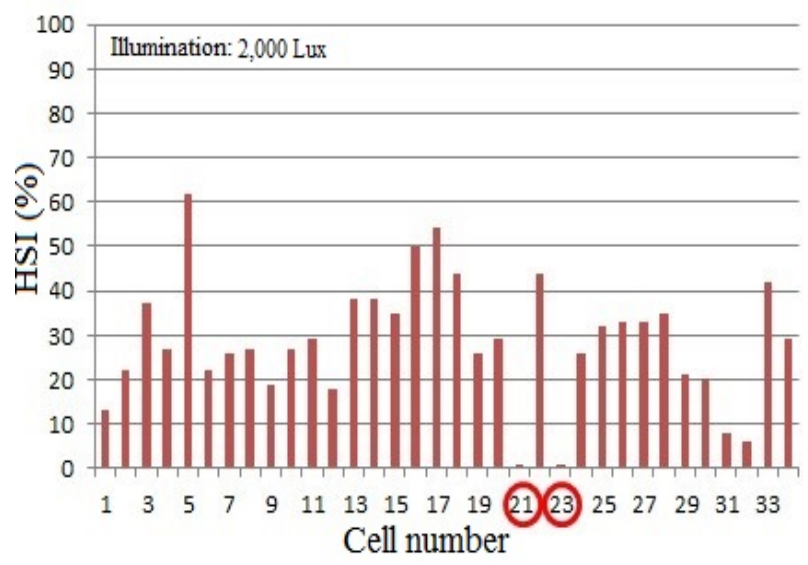

Fig. 15 Detection result at 2,000 Lux. 


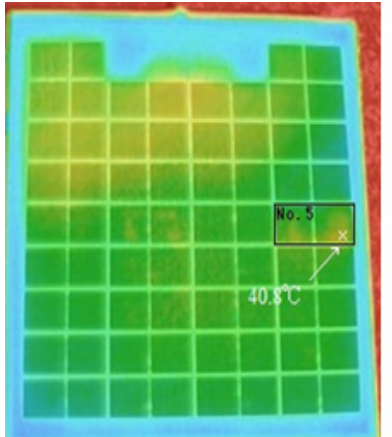

(a)

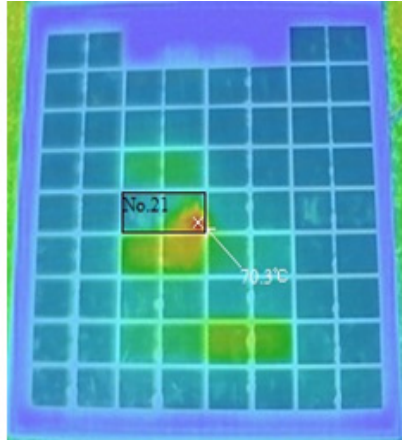

(b)
Fig. 16 IR image when No. 5 (Normal cell) and No. 21 (Defective cell) shaded.

From both Figs. 14 and 15, it can be seen that HSI of No. 21 and No. 23 cell is extremely low. In other word, cell No. 21 and No. 23 are high possibly crystal defective cells where hot-spot may arise. Moreover, due to the result of high illumination, No. 1, No. 31 and No. 32 cell is under a high risk of hot-spot arising. Since detection results under high and low illumination are consistent, it confirmed the effectiveness of voltage-based SRC detection method. Meanwhile, this result is also consistent with the result of current-based SRC detection under high illumination.

To further confirm the effectiveness of voltage-based method, we conduct an inspection experiment with infrared camera. In the voltage-based detection result in low illumination, HSI of No. 5 cell is relatively high which can be seen as a normal cell. Therefore, No. 5 and No. 21 of two cells are shaded respectively under the sunlight. The sunlight intensity is around $900-1,050 \mathrm{~W} / \mathrm{m}^{2}$ during the experiment. Fig. 16 indicates the IR images of these two cells.

From Fig. 16, the operating temperature of the normal cell is $40.8{ }^{\circ} \mathrm{C}$ while the mean temperature of rest of module is $39.8{ }^{\circ} \mathrm{C}$. In addition, the operating temperature of the hot-spot cell is $70.3{ }^{\circ} \mathrm{C}$ while the rest of the module is $40.2{ }^{\circ} \mathrm{C}$, which represents a temperature difference of $30.1{ }^{\circ} \mathrm{C}$. Moreover, No. 22 cell which is a normal cell is affected by No. 21, and rises to a high temperature. This further confirmed the validity of proposed method.

\section{Conclusions}

Aiming to develop a low cost and easy method to detect the hot-spot in PV module, this paper proposes a voltage-based SRC detection method for crystal defective cell in PV module before installation. The experiment confirms that, by calculating voltage HSI of cells, voltage-based SRC detection method is capable to avoid the problem of current-based SRC method and diagnoses hot-spot cells with defects effectively under low illumination.

Compared with conventional method, such as thermography, proposed detection system only consists of a PC, a projector, and a voltage sensor, the cost is greatly reduced. Furthermore, for each PV module, it only requires 2 or 3 minutes for inspection. Consequently, eliminating module with defect by proposed method previously, it is possible to greatly reduce the failure rate of solar array.

Since the experiment is for one module, future work discusses the effectiveness of voltage-based SRC detection on different kinds of module, and experiments will also be done on plural modules in the string to further verify the practicability of this method.

\section{References}

[1] Yasushi, O., Sanshiro, Y., and Daisuke, I. 2010. "An Investigation into Hot-Spot in PV Module." In Proceedings of the JSES/JWEA Joint Conference, 535-8.

[2] Moreton, R., Lorenzo, E., and Narvarte, L. 2014. "Dealing in Practice with Hot-Spots." In Proceedings of the 29th European Photovoltaic Solar Conference and Exhibition, 1-6.

[3] Bishop, J. W. 1989. "Microplasma Breakdown and Hot-Spots in Silicon Solar Cells." Solar Cell 26 (4): 335-49.

[4] Blake, F. A., and Hanson, K. L. 1969. "The Hot-Spot Failure Mode for Solar Arrays." In Proceedings of the 4th Intersociety Energy Conversion Engineering Conference, 575-81.

[5] Hoyer, U., Burkert, A., Auer, R., and Lutz, C. B. 2009. "Analysis of PV Modules by Electroluminescence and IR Thermography." In Proceedings of the 24th European Photovoltaic Solar Energy Conference and Exhibition, 
3262-6.

[6] Watanabe, S., Iijima, T., Ohya, H., Kudoh, T., and Itako, K. 2013. "Study on Temperature and Fundamental Characteristic of Hot-Spot of Single-Crystal Photovoltaic Cell by the Amount of Solar Radiation." In Proceedings of JSES/JWEA Joint Conference, 315-8.

[7] Kudou, H., Sugiyama, T., Kudoh, T., and Itako, K. 2013.
"Prototype of the Hot-Spot Rough-Diagnosis Equipment Using Projector for PV Module." In Proceedings of JSES/JWEA Joint Conference, 49-52.

[8] Itako, K., and Kudoh, T. 2014. "Study on Hotspot of a Single-crystal Photovoltaic Module." Journal of the Institute of Electrical Installation Engineers of Japan 34 (2): 140-6. 